\title{
Polaron in a quasi 1D cylindrical quantum wire
}

\author{
L.C.Fai ${ }^{1}$, V.Teboul ${ }^{2}$, A.Monteil $^{2}$, S.Maabou ${ }^{2}$, I.Nsangou $^{1}$ \\ 1 Department of Physics, Faculty of Science, \\ University of Dschang, Cameroon \\ 2 Laboratoire POMA, UMR CNRS 6136, \\ Université d'Angers, \\ 2 Bd Lavoisier, 49045 Angers, France
}

Received March 30, 2004

Polaron states in a quasi 1D cylindrical quantum wire with a parabolic confinement potential are investigated applying the Feynman variational principle. The effect of the wire radius on the polaron ground state energy level, the mass and the Fröhlich electron-phonon-coupling constant are obtained for the case of a quasi 1D cylindrical quantum wire. The effect of anisotropy of the structure on the polaron ground state energy level and the mass are also investigated.

It is observed that as the wire radius tends to zero, the polaron mass and energy diverge logarithmically. The polaron mass and energy differ from the canonical strong-coupling behavior by the Fröhlich electron-phonon coupling constant and the radius of the quasi 1D cylindrical quantum wire that are expressed through a logarithmic function. Moreover, it is observed that the polaron energy and mass for strong coupling for the case of the quasi 1D cylindrical quantum wire are greater than those for bulk crystals. It is also observed that the anisotropy of the structure considerably affects both the polaron ground state energy level and the mass. It is found that as the radius of the cylindrical wire reduces, the regimes of the weak and intermediate coupling polaron shorten while the region of the strong coupling polaron broadens and extends into those of the weak and intermediate ones.

Analytic expressions for the polaron ground state energy level and mass are derived for the case of strong coupling polarons.

Key words: polaron, polaron energy, polaron mass, parabolic confinement, Fröhlich electron-phonon coupling constant, quantum wire

PACS: 78.67.-n, 78.67.Hc, 71.38.-k 


\section{Introduction}

Recent developments in quasi 2D, 1D and 0D cylindrical quantum wire fabrication and in the fabrication of new electronic devices have stimulated the theoreticians' interest in formulating the models describing physical phenomena associated with cylindrical quantum wires [1-14]. Since the majority of nano-structures are ionic crystals and polar materials, polaronic effects may strongly effect their physical properties. The present paper investigates the effect of the wire radius on the polaron ground state energy level, the effective mass and the Fröhlich electronphonon-coupling constant for the case of a quasi 1D cylindrical quantum wire. In the cylindrical quantum wire configuration, the ultimate confinement effects quantize the carrier motion in the directions transverse to its length. Polaron energy is evaluated in [15-17] using perturbation theory, in [18] using the weak coupling approximation, in [19] using the dielectric continuum model and in [20] using the Feynman variational principle.

Landau was the first to predict the polaron concept [21]. It is studied in detail in $[21-27]$.

A polaron is a quasi particle that arises as a result of a conduction electron (or hole) together with its self-induced polarization in an ionic crystal or in a polar semiconductor [28]. To classify polarons, the Fröhlich electron-phonon coupling constant value $\alpha$ is a weak-coupling if $\alpha<1$, a strong-coupling if $\alpha \geqslant 7$ and an intermediate-coupling between these ranges. The majority of crystals are weak or intermediate-coupling polarons. Strong coupling is not attained even in strong ionic crystals such as alkaline halides. The polaron character is well pronounced only for strong coupling [29]. In nano-structures made of ionic crystals and polar materials the strong coupling can be realized at smaller $\alpha$ when the parabolic electronic confinement is introduced [30-34].

For cylindrical quantum wires it is possible to reduce the lower bound of the electron-phonon coupling constant's threshold value to within weak- or intermediatecoupling range. Quasi 1D cylindrical quantum wires are of interest as structures with maximal polaron effect. Here there exists at least one transport direction. It is obvious that these structures are quit promising in observing the strong coupling. When investigating the polaron problem in cylindrical quantum wires, it is necessary to consider both the electron and the phonon confinement. The electron confinement is described in [1,30-34] by means of a parabolic potential.

The investigation of the polaron states in a quasi-1D cylindrical quantum wire using Feynman variational principle results in the upper bound polaron ground state energy for arbitrary values of the Fröhlich electron-phonon coupling constant. Electron confinement is selected in the form of transverse parabolic potential since there are no rigid interface boundaries. Then we examine electron interaction only with 3D longitudinal polar optical phonons (3D-phonon approximation). For rigid interface boundaries, interface-like phonon modes are localized at the neighbourhood of a sharp boundary. There is also quantisation of bulk phonons. For the parabolic potential, interface-like phonon modes are rather smoothly distributed in space. For this 
we do the 3D-phonon approximation. Consequently, interface phonons may not be considered. This approach seems to be adequate since integral polaron effects result from the summation over all phonon spectra. In [34-37] interface-type longitudinal polar optical phonons have no contribution to polaron effects. The contribution of the interface-type phonons to the polaron energy is very much smaller than the contribution of the bulk-type phonon [19]. Bulk-type phonons play the dominant role in the polaron energy shift [38-47].

This work paves the way for investigating strong-coupling polarons in strong polar crystals. Thus we examine the polaron problem in the quasi 1D cylindrical quantum wire within the framework of the strong coupling theory. The model with parabolic confinement is preferable as it examines polaron states covering all values of Fröhlich electron-phonon coupling constant. The parabolic confinement potential is also introduced for technological reasons. At small values of the radius of the quantum wire the parabolic confinement potential can be regarded as a model for the real potential [48].

\section{Feynman variational principle}

The Feynman variational principle is one of the most effective methods when investigating the polaron problem for arbitrary values of the electron-phonon coupling constant, $\alpha$, and considers the exact and the model system. The action functional of the exact system is defined as:

$$
S[\vec{r}]=\int L \mathrm{~d} t
$$

and that of the model system is defined as:

$$
S_{0}[\vec{r}]=\int L_{0} \mathrm{~d} t
$$

where $\vec{r}$ is the radius vector, $t$ is the time, $L$ and $L_{0}$ are the Langrangians of the exact and model systems respectively.

The exact and the model action functionals are obtained as a result of the elimination of the phonon coordinates and the coordinates of the "fictitious" particles respectively. The statistical sum of the exact system is defined by

$$
Z=\operatorname{Sp} \int D \vec{r} \exp \{S[\vec{r}]\}
$$

and that of the model system is defined by

$$
Z_{0}=\operatorname{Sp} \int D \vec{r} \exp \left\{S_{0}[\vec{r}]\right\}
$$

Here $D \vec{r}$ denotes path integration and Sp the spur. 
In our evaluations, the statistical sum for the exact system is defined as $\tilde{Z}$ :

$$
\tilde{Z}=Z_{0}\left\langle\exp \left\{S[\vec{r}]-S_{0}[\vec{r}]\right\}\right\rangle .
$$

The angle brackets in (2.3) denote the averaging over electron paths and are defined as follows:

$$
\langle F[\vec{r}]\rangle=\frac{\operatorname{Sp} \int D \vec{r} F[\vec{r}] \exp \left\{S_{0}\right\}}{\operatorname{Sp} \int D \vec{r} \exp \left\{S_{0}\right\}} .
$$

The basis of Feynman variational method is the Jensen-Feynman inequality [28]:

$$
\left\langle\exp \left(S[\vec{r}]-S_{o}[\vec{r}]\right)\right\rangle \geqslant \exp \left\langle S[\vec{r}]-S_{o}[\vec{r}]\right\rangle .
$$

The Feynman's statistical sum $Z_{\mathrm{F}}$ is evaluated using (2.3) and (2.4):

$$
\ln \tilde{Z} \geqslant \ln Z_{\mathrm{F}} \equiv \ln Z_{o}-\left\langle S[\vec{r}]-S_{o}[\vec{r}]\right\rangle \text {. }
$$

We consider the total momentum $\vec{p}$ of the polaron to be the only continuous quantum number. The dependence of the energy on the momentum (for the case of an isotropic crystal) has the form:

$$
E=E_{0}(v)+p^{2} E_{2}(v)+p^{4} E_{4}(v)+\cdots .
$$

Here the quantities $E_{0}, E_{2}, E_{4}, \cdots$, are coefficients of expansion of (2.6) and $v$ is the totality of discrete quantum numbers of the system.

We evaluate the statistical sum for the system using (2.1) and limit ourselves only to the first two terms of the expansion as higher order terms for low temperatures are exponentially small (in the statistical sum expression) compared to the first two terms. If the polaron effective mass is defined by $M=1 / 2 E_{2}$ then it follows that

$$
\ln Z=\ln \left(\frac{V}{(2 \pi \hbar)^{3}}\left(\frac{2 \pi m_{\mathrm{e}}}{\lambda}\right)^{\frac{3}{2}}\right)-\lambda E_{0}+\frac{3}{2} \ln \left(\frac{M}{m_{\mathrm{e}}}\right), \quad \lambda \equiv \frac{1}{T} .
$$

Here $T$ is the absolute temperature. Comparing (2.5) and (2.7) we can obtain the expressions for the Feynman variational polaron energy $E$ and effective mass $M$. The polaron ground state energy is obtained as the coefficient of $\lambda$ in (2.7) and the polaron effective mass from the term independent of $\lambda$. We consider the case of low temperatures $T \rightarrow 0(\lambda \rightarrow \infty)$.

\section{Feynman polaron in a quasi 1D cylindrical quantum wire}

Consider the motion of an electron in a quasi 1D cylindrical quantum wire that has a transverse parabolic confinement potential. The electron motion in the direction of the axis of the cylindrical quantum wire (OZ-axis) is free. The Hamiltonian of the polaron problem has the form:

$$
\hat{H}=\hat{H}_{\mathrm{e}}+\hat{H}_{\mathrm{ph}}+\hat{H}_{\mathrm{e}-\mathrm{ph}}
$$


where $\hat{H}_{\mathrm{e}}$ is the electron Hamiltonian in cylindrical coordinates:

$$
\hat{H}_{\mathrm{e}}=\frac{\hat{P}_{\perp}^{2}}{2 m_{\perp}}+\frac{\hat{P}_{\|}^{2}}{2 m_{\|}}+\frac{m_{\perp} \Omega^{2}}{2} \rho^{2} .
$$

Here $\rho^{2}=x^{2}+y^{2} ; \hat{P}_{\perp}, m_{\perp}$ and $\hat{P}_{\|}, m_{\|}$components of the operator of the momentum and electron band mass in the transversal and longitudinal directions respectively; $\Omega$ is the frequency characterizing the parabolic confinement potential; and

$$
\left\{\begin{array}{l}
\hat{H}_{\mathrm{ph}}=\sum_{\vec{q}} \hbar \omega_{\vec{q}} \hat{b}_{\vec{q}}^{+} \hat{b}_{\vec{q}}, \\
\hat{H}_{\mathrm{e}-\mathrm{ph}}=\sum_{\vec{q}}\left[\gamma_{\vec{q}} \hat{b}_{\vec{q}} \mathrm{e}^{\mathrm{i} \vec{q} \vec{r}}+\gamma_{\vec{q}}^{*} \hat{b}_{\vec{q}}^{+} \mathrm{e}^{-\mathrm{i} \vec{q} \vec{r}}\right]
\end{array}\right.
$$

are respectively the phonon contribution and electron-phonon interaction Hamiltonians. Here $\gamma_{\vec{q}}$ is the amplitude of the electron-phonon interaction and $\omega_{\vec{q}}$ is the phonon frequency numbered by the wave vector $\vec{q}$ :

$$
\gamma_{\vec{q}}=\left[\frac{\alpha \pi}{V}\left(\frac{\hbar \omega_{o}}{q}\right)^{2} R_{\mathrm{p}}\right]^{\frac{1}{2}}, \quad R_{\mathrm{p}}=\left(\frac{\hbar}{2 m_{\mathrm{e}} \omega_{o}}\right)^{\frac{1}{2}}, \quad q^{2}=q_{\perp}^{2}+q_{\|}^{2},
$$

$\omega_{0}$ is the dispersionless phonon frequency and $R_{\mathrm{p}}$ is the polaron radius. $\hat{b}_{\vec{q}}^{+}$and $\hat{b}_{\vec{q}}$ are the creation and annihilation operators respectively.

Considering the Hamiltonian (3.1), the model Lagrangian $L_{0}$ for the system (considering the ground state) is selected in the one - oscillatory approximation:

$$
L_{0}=-\frac{m_{\perp} \dot{\rho}^{2}}{2 \hbar^{2}}-\frac{m_{\|} \dot{z}^{2}}{2 \hbar^{2}}-\frac{m_{\perp} \Omega^{2}}{2} \rho^{2}-\frac{M_{\perp} \dot{R}^{2}}{2 \hbar^{2}}-\frac{M_{\|} \dot{Z}^{2}}{2 \hbar^{2}}-\frac{\kappa_{\perp}}{2}(\vec{R}-\vec{\rho})^{2}-\frac{\kappa_{\|}}{2}\left(\vec{Z}^{\prime}-\vec{Z}\right)^{2},
$$

where $R$ and $Z$ are coordinates of the model particle; $\rho$ and $z$ are coordinates of the electron, and $m_{\perp}$ and $m_{\|}$are the components of the electron masses in the oxyplane and in the $o z$-direction respectively. The quantities $M_{\perp}, M_{\|}, \kappa_{\perp}$ and $\kappa_{\|}$serve as variational parameters, while $\omega_{\mathrm{f}}$ is the elastic coupling frequency.

From the Lagrangian in (3.3), the transverse and longitudinal equations of motion for the model system are independent. The model Lagrangian in (3.3) simulates the interacting electron-phonon system. Considering the form of the model Lagrangian, the equations of motion along the transversal and longitudinal directions are mutually independent. From the equation of motion considering the transversal direction, the following eigen frequencies are obtained:

$$
\omega_{\perp 1,2}^{2}=\frac{1}{2}\left(\frac{\kappa_{\perp}}{\mu_{\perp}}+\Omega^{2}\right) \pm \frac{1}{2}\left[\left(\frac{\kappa_{\perp}}{\mu_{\perp}}+\Omega^{2}\right)^{2}-\frac{4 \kappa_{\perp} \Omega^{2}}{M_{\perp}}\right]^{\frac{1}{2}}, \quad \frac{1}{\mu_{\perp}}=\frac{1}{m_{\perp}}+\frac{1}{M_{\perp}} .
$$

In order to diagonalize the Lagrangian (3.3) we do the linear transformation of the variables $R$ and $\rho$ using the modes in (3.4):

$$
\left\{\begin{array}{l}
\vec{\rho}=c_{1} \vec{\xi}_{1}\left(\omega_{\perp 1}\right)+c_{2} F_{2}\left(\omega_{\perp 2}\right) \vec{\xi}_{2}\left(\omega_{\perp 2}\right) \\
\vec{R}=c_{1} F_{1}\left(\omega_{\perp 1}\right) \vec{\xi}_{1}\left(\omega_{\perp 1}\right)+c_{2} \vec{\xi}_{2}\left(\omega_{\perp 2}\right)
\end{array}\right.
$$


Here

$$
\begin{array}{ll}
F_{1}\left(\omega_{\perp 1}\right)=\frac{\kappa_{\perp}}{-M_{\perp} \omega_{\perp 1}^{2}+\kappa_{\perp}}, & F_{2}\left(\omega_{\perp 2}\right)=-\frac{M_{\perp}}{m_{\perp}} F_{1}\left(\omega_{\perp 1}\right), \\
c_{1}^{2}=\frac{1}{m_{\perp}+M_{\perp} F_{1}^{2}}, & c_{2}^{2}=\frac{m_{\perp}}{M_{\perp}} c_{1}^{2} .
\end{array}
$$

Also

$$
\left\{\begin{array}{llll}
\rho_{1}=Z-z, & \rho_{2}=\frac{m_{\|} z+M_{\|} Z}{M}, & M=m_{\|}+M_{\|}, & \nu^{2}=u^{2} \omega_{\| f} \\
u^{2}=\frac{M_{\|}}{m_{\|}}, & \mu_{\|}=\frac{m_{\|} M_{\|}}{M}, & a_{1}+a_{2}=1, & a_{1}=1 / u^{2} .
\end{array}\right.
$$

It may be seen from this transformation that on the axis of the wire there is one free and one oscillatory polaron motion. Considering the transformation of variables and (2.1), (2.2), (2.4) and (3.3) to (3.5) we obtain the expression:

$$
\begin{aligned}
\left\langle S-S_{\perp 0}-S_{\| 0}\right\rangle= & 2 \ln \left(2 \sinh \frac{\lambda \hbar \omega_{\perp 1}}{2}\right)+2 \ln \left(2 \sinh \frac{\lambda \hbar \omega_{\perp 2}}{2}\right) \\
& +\frac{\hbar \kappa_{\perp}}{2} \int_{0}^{\lambda}\left\langle\rho^{2}\right\rangle d \tau+\frac{\hbar \kappa_{\|}}{2} \int_{0}^{\lambda}\left\langle z^{2}\right\rangle d \tau-\left\langle\Phi_{\omega_{\perp f}}\right\rangle-\left\langle\Phi_{\omega_{\| f}}\right\rangle+\langle\Phi\rangle .
\end{aligned}
$$

Here

$$
\begin{aligned}
\left\langle\Phi_{\omega_{\perp f}}\right\rangle & =\frac{\hbar \kappa_{\perp}^{2}}{4 M_{\perp} \omega_{\perp f}} \int_{0}^{\lambda} \int_{0}^{\lambda}\left\langle\rho_{\tau} \rho_{\sigma}\right\rangle F\left(\omega_{\perp f}\right) \mathrm{d} \tau \mathrm{d} \sigma \\
\left\langle\Phi_{\omega_{\perp f}}\right\rangle & =\frac{\hbar \kappa_{\|}^{2}}{4 M_{\|} \omega_{\| f}} \int_{0}^{\lambda} \int_{0}^{\lambda}\left\langle z_{\tau} z_{\sigma}\right\rangle F\left(\omega_{\| f}\right) \mathrm{d} \tau \mathrm{d} \sigma \\
\langle\Phi\rangle & =\alpha \lambda \int_{0}^{\infty} F\left(A_{\perp}, A_{\|}\right) \mathrm{e}^{-\tau} \mathrm{d} \tau
\end{aligned}
$$

and

$$
\begin{gathered}
F\left(A_{\perp}, A_{\|}\right)=\beta \sqrt{\frac{1}{\pi}} \frac{1}{\left(\left|\varepsilon A_{\|}-A_{\perp}\right|\right)^{1 / 2}} \begin{cases}A r \sinh (A-1)^{1 / 2}, & A>1, \\
\arcsin (1-A)^{1 / 2}, & A<1,\end{cases} \\
A=\varepsilon \frac{A_{\|}}{A_{\perp}}, \\
A_{\perp}=\sum_{j=1}^{2} \frac{a_{j}}{\omega_{\perp j}}\left(1-\mathrm{e}^{-\omega_{\perp j} \tau}\right), \quad a_{1}=\frac{\omega_{1}^{2}-\omega_{\mathrm{f}}^{2}}{\omega_{1}^{2}-\omega_{2}^{2}}, \quad a_{2}=\frac{\omega_{\mathrm{f}}^{2}-\omega_{2}^{2}}{\omega_{1}^{2}-\omega_{2}^{2}}, \\
A_{\|}=\frac{a_{2}}{\nu}\left(1-\mathrm{e}^{-\nu \tau}\right)+a_{1} \tau,
\end{gathered}
$$




$$
F(\omega)=\frac{\cosh \left(\hbar \omega\left[|\tau-\sigma|-\frac{\lambda}{2}\right]\right)}{\sinh (\lambda \hbar \omega / 2)} .
$$

We evaluate $\left\langle\rho_{\tau}^{2}\right\rangle,\left\langle\rho_{\tau} \rho_{\sigma}\right\rangle$ and $\left\langle z_{\tau}^{2}\right\rangle,\left\langle z_{\tau} z_{\sigma}\right\rangle$ respectively using the productive functions

$$
\Psi_{\perp}(\varsigma, \eta) \equiv \exp \{-\Sigma-\Xi\}
$$

where

$$
\begin{aligned}
& \Sigma=\frac{\hbar c_{1}^{2} q_{\perp}^{2}}{4 \omega_{\perp 1}}\left[\left(\varsigma^{2}+\eta^{2}\right) \operatorname{coth}\left(\frac{\lambda \hbar \omega_{\perp 1}}{2}\right)-2 \varsigma \eta F\left(\omega_{\perp 1}\right)\right], \\
& \Xi=\frac{\hbar c_{2}^{2} F_{2}^{2} q_{\perp}^{2}}{4 \omega_{\perp 2}}\left[\left(\varsigma^{2}+\eta^{2}\right) \operatorname{coth}\left(\frac{\lambda \hbar \omega_{\perp 2}}{2}\right)-2 \varsigma \eta F\left(\omega_{\perp 2}\right)\right]
\end{aligned}
$$

and

$$
\Psi_{\|}(\varsigma, \eta) \equiv \exp \{-\Lambda-\Upsilon\}
$$

where

$$
\begin{aligned}
\Lambda & =\frac{\hbar c_{1}^{2} q_{\perp}^{2}}{4 \omega_{\perp 1}}\left[\left(\varsigma^{2}+\eta^{2}\right) \operatorname{coth}\left(\frac{\lambda \hbar \omega_{\perp 1}}{2}\right)-2 \varsigma \eta F\left(\omega_{\perp 1}\right)\right], \\
\Upsilon & =\frac{\hbar c_{2}^{2} F_{2}^{2} q_{\perp}^{2}}{4 \omega_{\perp 2}}\left[\left(\varsigma^{2}+\eta^{2}\right) \operatorname{coth}\left(\frac{\lambda \hbar \omega_{\perp 2}}{2}\right)-2 \varsigma \eta F\left(\omega_{\perp 2}\right)\right] .
\end{aligned}
$$

From considering (2.5) and (2.7), for low temperatures $T \rightarrow 0(\lambda \rightarrow \infty)$ and from the fact that $\varsigma=\eta= \pm 1$ we obtain the polaron energy and mass. In further evaluations of the polaron energy and mass, the Feynman's units [25] are used: $\hbar \omega_{0}$ for energy and $R_{\mathrm{p}}$ for length. The Feynman polaron dimensionless variational energy is found to be:

$$
E=\frac{\nu}{4}\left(1-\frac{1}{u}\right)^{2}+\Omega+\frac{\left(\Omega-\omega_{\perp 1}\right)^{2}\left(\Omega-\omega_{\perp 2}\right)^{2}}{\Omega^{2}\left(\omega_{\perp 1}+\omega_{\perp 2}\right)}-\alpha \int_{0}^{\infty} F\left(A_{\perp}, A_{\|}\right) \mathrm{e}^{-\tau} \mathrm{d} \tau
$$

and the dimensionless variational polaron mass may be conveniently evaluated through the expression

$$
M=u^{2}+\frac{\left(\omega_{\perp 1}^{2}-\Omega^{2}\right)\left(\Omega^{2}-\omega_{\perp 2}^{2}\right)}{\omega_{\perp 1}^{2} \omega_{\perp 2}^{2}}-1 .
$$

In (3.6), $\varepsilon$ is the parameter characterizing the anisotropy of the structure bulk.

The polaron energy is found by minimizing the polaron variational energy in (3.6) and the mass by substituting in the expression of the mass in (3.7) the threshold values of $\nu, u, \omega_{\perp 1}$ and $\omega_{\perp 2}$ for which the energy is minimum. The numerical results are shown in the figures $1-4$ below. In the figures 1 and 3 the absolute value of the polaron energy is considered for convenience to aid easy analysis.

From expressions (3.6) and (3.7) the analytic dimensionless expressions for the polaron energy and effective mass for the strong-coupling polarons are obtained respectively as:

$$
E=-\frac{4 \alpha^{2} \beta^{2}}{\varepsilon \pi} \ln ^{2}\left(\frac{\varepsilon \sqrt{\pi}}{2 \mathrm{e} \beta \alpha R f(Q)}\right)-\frac{2}{R^{2}}
$$




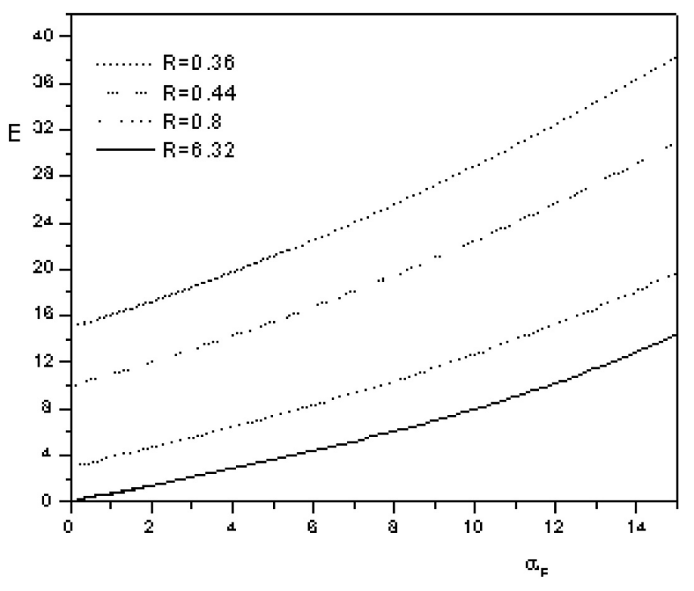

Figure 1. Polaron energy versus Fröhlich constant for different radii.

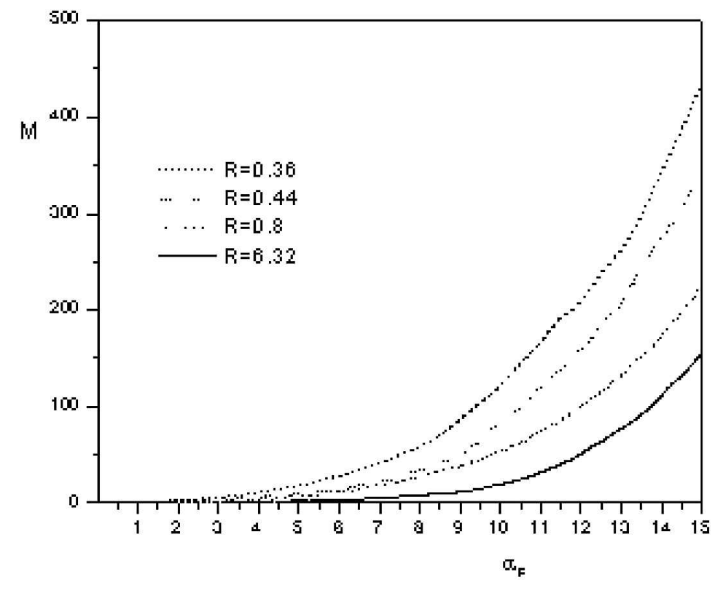

Figure 2. Polaron mass versus Fröhlich constant for different radii.

and

$$
M=\frac{256 \alpha^{4} \beta^{4}}{\varepsilon^{2} \pi^{2}} \ln ^{2}\left(\frac{\varepsilon \sqrt{\pi}}{2 \mathrm{e} \beta \alpha R f(Q)}\right)
$$

Here

$$
\begin{gathered}
f(Q)=-\ln [-Q \ln [-Q \ln \ldots \ln Q]], \quad Q=\frac{2 \mathrm{e} \beta R \alpha}{\varepsilon \sqrt{\pi}}, \\
\mathrm{e}=2.718281828
\end{gathered}
$$

and

$$
R=\left(\frac{2 \omega_{0}}{\Omega}\right)^{1 / 2}
$$

is the radius of the quasi $1 \mathrm{D}$ cylindrical quantum wire.

Rigorous weak-coupling expansion for the polaron ground state energy and effective mass yield [25]:

$$
E=-\alpha-0.01592 \alpha^{2}-\cdots, \quad M=1+\frac{\alpha}{6}+0.02363 \alpha^{2}+\cdots .
$$

The expansion for the strong-coupling polaron yields [26]:

$$
E=-0.109 \alpha^{2}, \quad M=0.02 \alpha^{4} .
$$

Polaron excitation in $[49,50]$ for the strong-coupling polaron in a $3 \mathrm{D}$ structure yields the energy:

$$
E=-\frac{\alpha^{2}}{9 \pi} .
$$

Since

$$
\ln ^{2}\left(\frac{\varepsilon \sqrt{\pi}}{2 \mathrm{e} \alpha \beta R f(Q)}\right) \geqslant 1
$$




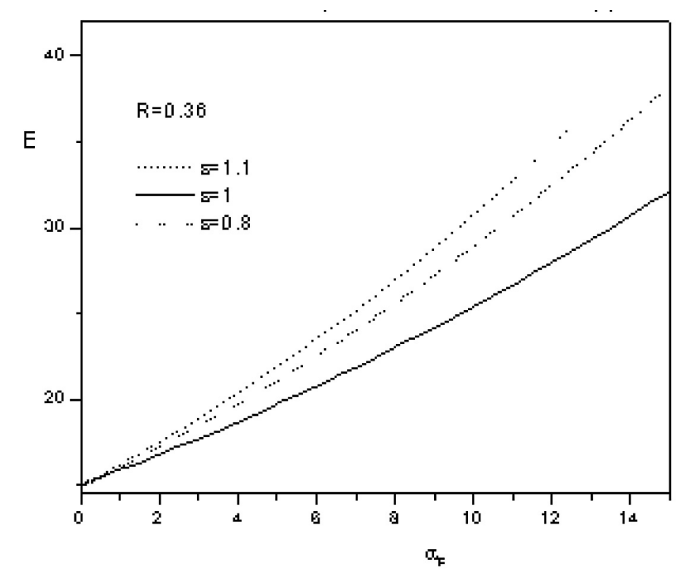

Figure 3. Polaron energy versus Fröhlich constant for different parameters of the anisotropy.

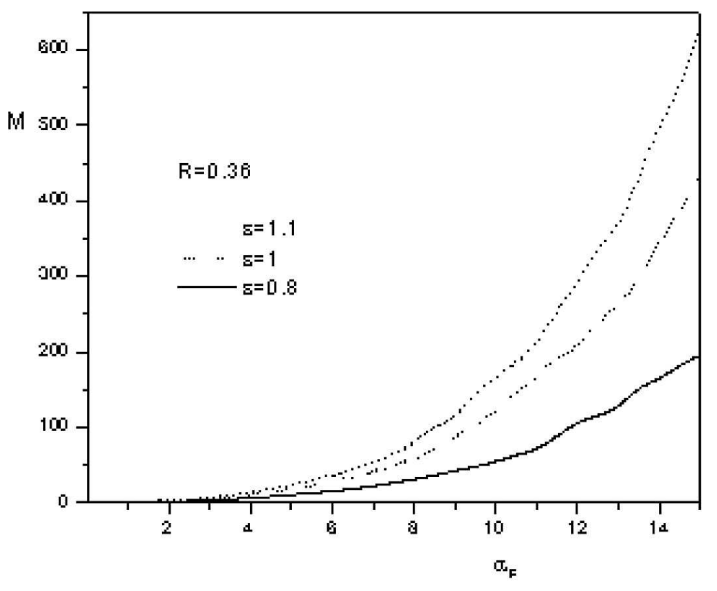

Figure 4. Polaron mass versus Fröhlich constant for different parameters of the anisotropy.

then from the above results, the polaron ground state energy and mass in a quasi 1D cylindrical quantum wire are greater than those in 3D structures. It is also seen that the decrease in the radius of the structure leads to the increase in polaron ground state energy and in the effective mass. It follows that the confinement leads to an enhancement of the electron-phonon interaction.

\section{Conclusions}

Figures 1 and 2 are respectively the plots of the polaron energy and mass versus Fröhlich electron-phonon coupling constant for different structure radii. It is observed that as the wire radius tends to zero, the polaron mass and energy diverge logarithmically and the region of strong-coupling polaron is shifted to those of weakand intermediate-coupling in which the energy, $E=$ const $\times \alpha$. This shows that as $R \rightarrow 0$ the weak and intermediate regions vanish. The behaviors in figures 1 and 2 differ from those in [51-54] only in their logarithmic dependence. Our results confirm those in $[33,48,54]$ in that the polaron energy and mass increase with a decrease of a radius of the cylindrical quantum wire. It follows that the confinement leads to the enhancement of the electron-phonon interaction. This is also confirmed by $[23,25,27,41]$. The difference in these results and those in [53] is that the polaron energy and mass pass through a maximum (in their plot against the coupling constant) and then decreases to bulk polaron values. For our case due to the strong confinement, the regions of weak and intermediate polarons disappear. The results in [17] show that the polaron energy and mass decrease through a minimum in their plot against the radius and then increase to a constant value. In our opinion this minimum should not be expected. This may imply that the bulk optical polaron is bound to a Coulomb center. Therefore the pertubative method may not be appropriate for the treatment of strong coupling polarons. 
Our results show that the polaron mass and energy differ from the canonical strong-coupling behavior by the Fröhlich electron-phonon coupling constant and the radius of the quasi $1 \mathrm{D}$ cylindrical quantum wire that are expressed through a logarithmic function. It is observed that the polaron energy and mass for strong coupling for the case of the quasi 1D cylindrical quantum wire are greater than those for bulk structures. This is in agreement with [34]. From the analytic expressions of the energy and mass, since

$$
\ln ^{2}\left(\frac{\varepsilon \sqrt{\pi}}{2 \mathrm{e} \alpha \beta R f(Q)}\right) \geqslant 1
$$

it follows that the polaron ground state energy and mass in a quasi 1D cylindrical quantum wire are greater than those in 3D structures. If we compare our results with those derived from the strong coupling theory we see that ours yield significantly improved energy upper bounds.

It is also observed that the decrease of the radius of the structure favors strongcoupling polaron for smaller Fröhlich electron-phonon coupling constant. Thus to enhance a strong-coupling polaron it is sufficient to reduce the radius of the structure. From our results it is seen that the parameters $\alpha$ and $R$ control the polaron state in an interrelated manner. They vary in an interrelated manner in favor of a strong coupling polaron. It is observed that the Fröhlich electron-phonon-coupling constant for strong-coupling in the case of the quantum wire is found in the weak and intermediate-coupling range.

Figures 3 and 4, show the effect of the anisotropy on the polaron energy and mass respectively. It is also observed that the anisotropy of the structure considerably affects the polaron ground state energy level and the mass.

\section{References}

1. Hudgins R.R., Durourd P., Tenenbaum J.M., Jarrold M.F., Phys. Rev. Lett., 1997, $\mathbf{7 8}, 4213$.

2. Zhao Q.X., Magnea N., Pautrat J.L., Phys. Rev. B, 1995, 52, 16612.

3. Lucsak F., Brosens F., Devreese J.T., Phys. Rev. B, 1995, 52, No. 17, 12743.

4. Nagamune Y., Arakawa Y., Tsukamoto S., Nishioka M., Sasaki S., Mikra N., Phys. Rev. Lett., 1993, 69, 2963.

5. Osorio A.P., Phys. Rev. B, 1995, 52, No. 7, 4662.

6. Osorio F.A.P., Degani M.H., Hipolito' O., Phys. Rev. B, 1998, 37, No. 3, 1402.

7. Dneprovskii V., Gushina N., Pavlov O., Poborchii V., Salamatina I., Zhukov E., Phys. Lett. A, 1995, 204, 59.

8. Brandbyge M., Schiötz J., Sörensen M.R., Stoltze P., Jacobsen K.W., Nörskov J.K., Olesen L., Laegsgaard E., Stensgaard I., Besenbacher F., Phys. Rev. B, 1995, 52, 8499.

9. Temkin H., Dolan G.J., Parish M.B., Chu S.N.G., Appl. Phys. Lett., 1997, 40, 413.

10. Tonucci R.C., Justus B.L., Campillo A.J., Ford C.E., Science, 1992, 258, 783.

11. Watt M., Sotomayer-Torres C.M., Arnot H.E.G., Beaumont S.P., Semicond. Sci. Technol., 1990, 5, 285.

12. Duan J., Bishops G.G., Gillman E.S., Chern G., Safron S.A., Skofronick J.G., Vac. Sci. Technol. A, 1992, 10, 1999. 
13. Dominguez-Adame F., Gomez I., Orellana P.A., Ladron de Guevana M.L., Micoelectronics Journal, 2004, 35, 87.

14. Orellana P.A., Dominguez-Adame F., Gomez I., Ladron de Guevana M.L., Phys. Rev. B, 2003, 67, 085321.

15. Pokatilov E.P., Klimin S.N., Balaban S.N., Fomin V.M., Phys. Stat. Sol. B, 1995, 189, 433.

16. Pokatilov E.P., Fomin V.M., Balaban S.N., Klimin S.N., Devreese J.T., Phys Stat. Sol. B, 1998, 210, 879.

17. Erçelebi A., Senger R.T., Phys. Rev. B, 1996, 53, No. 16, 11008.

18. Klimin S.N., Pokatilov E.P., Fomin V.M., Phys Stat. Sol. B, 1994, 184, 373.

19. Kazunori Oshiro, Koji Akai, Mitsuru Matsuura, Phys Rev. B, 1998, 58, No. 12, 7986.

20. Senger R.T., Erçelebi A., J. Phys.: Cond. Matt., 1997, 9, 5067.

21. Landua L.D., Phys. Sowjetunion Z., 1993, 3, 664.

22. Pekar S.I., Zh. Eksp. Teor. Fiz., 1946, 16, 335.

23. Feynman R.P., Phys. Rev., 1955, 97, 660.

24. Pokatilov E.P., Fomin V.M., Beril S.I. Vibrational Excitations, Polarons and excitons in Multilayer Structures. Stiintsa, Kishinev, 1990.

25. Devreese J.T. Encyclopedia of Applied Physics, 14, 383. VCH Publishers, 1996.

26. Alexandrov A.S. Sir N. Mott. World Scientific Publishing Co Pte Ltd, p. 9, 1995.

27. Fröhlich H., Adv. Phys., 1954, 3, 325.

28. Devreese J.T. - In: Proc. of the International School of physics "Enrico Fermi" Course CXXXVI. G.Iodonisi, J.R. Schrieffer and M.L. Chiotalo (Eds.). IOSS Press, Amsterdam, 1998.

29. Pekar S.I. Research on Electron Theory In Crystals. U.S. ACE, Washington DC, 1963.

30. Pokatilov E.P., Fomin V.M., Klimin S.N., Balaban S.N., Fai L.C., Devreese J.T., Superlattices Microstructures, 1998, 23, 331.

31. Pokatilov E.P., Fomin V.M., Klimin S.N., Balaban S.N., Fai L.C. - In: $15^{\text {th }}$ General conf. of the Condensed Matter Division of the EPS. Baveno-Stresa, Italy, 22-25 April, 1996. Europhysics Conf. Abstracts, vol. 20a. EPS, Pavia, 48, 1996.

32. Pokatilov E.P., Fomin V.M., Klimin S.N., Devreese J.T., Balaban S.N., Fai L.C. - In: Proc. $9^{\text {th }}$ Int. Conf. on the Superlattices, Microstructures and Microdevices. Liege, 14-19, July, 1996. Abstract Workbook. ThP-77, 1996.

33. Fai L.C., Teboul V., Monteil A., Nsangou I., Maabou S., Condens. Matter Phys., 2004, 7, No. 1, 37.

34. Fai L.C., Nsangou I., Tchitnga R., Tchassem D. - In: ISSP International Workshop. Kashiwa-Japan, August 13-21, 2003. Abstract Workbook. 19P-13, 2003.

35. Devreese J.T., Fomin V.M., Gladilin V.N., Imanaka Y., Miura, Journal of Crystal Growth, 2000, 214/215, 465.

36. Pan J.S., Pan H.B., Phys. Stat. Sol., 1988, 148, 129.

37. Marini J.C., Srebe B., Kartheuser E., Phys. Rev. B, 1994, 50, 14302.

38. Klimin S.N., Pokatilov E.P., Fomin V.M., Phys. Stat. Sol. B, 1994, 184, 373.

39. Tanatar B., J. Phys. Condens. Matter, 1993, 5, 2203.

40. Degani M.H., Farias G.A., Phys. Rev. B, 1990, 42, 11950.

41. Constantinou N.C., Ridley B.K., J. Phys. Condens. Matter, 1989, 1, 2283.

42. Yildirim T., Erçelebi A., J. Phys. Condens. Matter, 1991, 3, 4357.

43. Yildirim T., Erçelebi A., J. Phys. Condens. Matter, 1991, 3, 1271.

44. Zhou H.Y., Gu S.W., Solid State Commun., 1994, 91, 725. 
45. Degani M.H., Hipolito O., Solid Sate Commun., 1998, 65, 1185.

46. Leburton J.P., J. Appl. Phys., 1984, 56, 2850.

47. Devreese J., Evrard R., Kartheuser E., Brosens F., Solid State Commun., 1982, 44, 1435.

48. Pokatilov E.P., Fomin V.M., Devreese J.T., Balaban S.N., Klimin S.N., Physica E, 1999, 4, 156.

49. Zhu K.D., Gu S.W., Phys. Letters A, 1992, 171, 113.

50. Haupt R., Wendler L., Ann. Phys. (USA), 1994, 233, 214.

51. Erçelebi A., Senger R.T., Solid State Commun., 19996, 97, No. 6, 509.

52. Erçelebi A., Senger R.T., Phys. Rev. B, 1996, 53, No. 16, 11008.

53. Senger R.T., Erçelebi A., J. Phys: Cond. Matt., 1997, 9, 5067.

54. Matos-Abiague A., Semicond. Sci. Technol., 2002, 17, 150.

\title{
Полярон у квазіодновимірному циліндричному квантовому дроті
}

\author{
Л.Фаї ${ }^{1}$, В.Тебул ${ }^{2}$, А.Монтейл ${ }^{2}$, С.Маабу ${ }^{2}$, І.Нсангу ${ }^{1}$ \\ 1 Факультет природничих наук, \\ Університет м. Джанг, Камерун \\ 2 UMR CNRS 6136, \\ Університет м. Анже, \\ вул. Лавуаз'є, Анже 49045, Франція
}

\section{Отримано 30 березня 2004 р.}

За допомогою варіаційного принципу Фейнмана вивчаються стани полярона в квазіодновимірному циліндричному квантовому дроті 3 параболічним обмеженим потенціалом. Досліджується вплив радіуса дроту на енергетичний рівень основного стану полярона, масу і електрон-фонон постійну зв'язку Фрьоліха. Також вивчається ефект анізотропії структури на енергетичний рівень основного стану полярона і його масу. Виявлено, що якщо радіус дроту прямує до нуля, маса і енергія полярона розбігаються логарифмічно. Маса і енергія полярона відрізняються від канонічної поведінки сильного зв'язку на електрон-фонон постійну зв'язку Фрьоліха і на радіус квазіодновимірного циліндричного квантового дроту, які виражаються через логарифмічну функцію. Більше того, спостережено, що енергія та маса полярона для випадку квазіодновимірного циліндричного кванто-вого дроту є більшими, ніж для випадку об'ємних кристалів. Також виявлено, що анізотропія структури сильно впливає на енергетичний рівень основного стану полярона та його масу. Знайдено, що якщо радіус циліндричного дроту зменшується, режими слабкого та середнього зв'язку полярона скорочуються, тоді як область сильного зв'язку полярона розширюється. Отримано аналітичні вирази для енергетичного рівня основного стану полярона і для його маси для випадку сильного зв'язку поляронів.

Ключові слова: полярон, енергія полярона, маса полярона, електрон-фонон постійна зв'язку Фрьоліха, квантовий дріт

PACS: 78.67.-n, 78.67.Hc, 71.38.-k 